\title{
PMD-Insensitive SCM Optical Receiver Using Polarization Diversity
}

\author{
Rongqing Hui, Senior Member, IEEE, Christopher Allen, Senior Member, IEEE, and \\ Kenneth Demarest, Senior Member, IEEE
}

\begin{abstract}
Subcarrier multiplexing (SCM) optical systems with high subcarrier frequencies are susceptible to power fading caused by fiber polarization-mode dispersion (PMD). In this letter, an SCM optical receiver free of carrier fading is proposed and demonstrated using polarization diversity. Unlike conventional PMD compensators, this setup does not require a tunable optical delay line.
\end{abstract}

Index Terms-Optical communication, optical modulation, polarization, polarization-mode dispersion, subcarrier multiplexing (SCM).

$\mathbf{I}$ $\mathrm{N}$ HIGH-SPEED long-distance optical transmission systems using subcarrier multiplexing (SCM), to minimize the impact of fiber chromatic dispersion, optical single-sideband (SSB) modulation has been used, which also increases the spectral efficiency [1]. For this case, system tolerance to chromatic dispersion depends on the data rate on each individual subcarrier channel. However, polarization-mode dispersion (PMD) may become a limiting factor in this type of optical system.

In an optical fiber with PMD, two distinct orthogonal polarization modes exist with different propagation constants and different group velocities. This is described as the differential group delay (DGD) between the two orthogonal principal states of polarization (PSPs) of the fiber. Due to the statistical nature of the perturbations along the fiber, instantaneous DGD has a random value that generally follows a Maxwellian probability distribution. While SCM optical modulation distributes the total capacity of each laser transmitter into a number of subcarriers, and therefore, the data rate carried by each subcarrier is relatively low, the impact of PMD on SCM systems is determined mainly by the frequency of each RF subcarrier, rather than by the bandwidth of each individual subcarrier.

If we assume that the RF frequency of a subcarrier is $\Delta \omega$, this will also be the frequency separation between the carrier and the subcarrier in the optical domain. During fiber transmission, both the carrier and the subcarrier are decomposed into fast and slow PSPs. This causes a PMD-induced signal fading if the fiber DGD is sufficiently high. To illustrate this in a simple way, we assume that the optical field of both carrier and subcarrier are equally split into the fast and the slow PSPs and denote $E_{c x}, E_{c y}, E_{s x}$, and $E_{s y}$ as carrier and subcarrier optical field

Manuscript received May 20, 2002; revised August 7, 2002. This work was supported by Sprint Communications Company LP.

The authors are with the Information and Telecommunication Technology Center, Department of Electrical Engineering and Computer Science, University of Kansas, Lawrence, KS 66044 USA.

Digital Object Identifier 10.1109/LPT.2002.803900 components on the fast $(x)$ and slow (y) PSP, respectively. At the receiver photodiode, the optical carrier beats with the optical subcarrier creating two photocurrent components: $i_{x}(t)=$ $\left|E_{c x} E_{s x}\right| \cos (\Delta \omega t)$ and $i_{y}(t)=\left|E_{c y} E_{s y}\right| \cos [\Delta \omega(t+\Delta \tau)]$, where $\Delta \tau$ is the relative propagation delay between the fast and the slow PSPs, i.e., DGD. Therefore, the total received subcarrier component in the RF domain is

$$
I(t)=\left|E_{c x} E_{s x}\right| \cos (\Delta \omega t)+\left|E_{c y} E_{s y}\right| \cos (\Delta \omega t+\Delta \omega \Delta \tau) .
$$

Because of the assumption of equal power splitting, $a(t)=$ $\left|E_{c x} E_{s x}\right|=\left|E_{c y} E_{s y}\right|$, and we have

$$
I(t)=2 a(t) A(\Delta \tau) \cos [\Delta \omega(t+\Delta \tau / 2)] .
$$

The term $a(t)$ represents the digital data carried by the subcarrier, $\cos [\Delta \omega(t+\Delta \tau / 2)]$ is the recovered RF subcarrier with a phase shift $\Delta \omega \Delta \tau / 2$, and $A(\Delta \tau)=\cos (\Delta \omega \cdot \Delta \tau / 2) \leq 1$ represents the PMD-induced subcarrier fading. A complete fading happens when $\Delta \tau=\pi / \Delta \omega$. A complete signal fading occurs in this case because $\Delta \tau=\Delta T / 2$ where $\Delta T$ is the period of the subcarrier. PMD-induced carrier fading happens to both double-sideband and single-sideband modulated optical SCM signals [2], and it is indeed one of the biggest problems preventing long-distance, high-capacity applications of optical SCM systems. For an SCM system with the highest subcarrier frequency of $20 \mathrm{GHz}$, although the data rate on the subcarrier may be low, the accumulated DGD in the transmission fiber has to be much smaller than $25 \mathrm{ps}$ in order to avoid carrier fading. Therefore, for most practical applications of reasonable transmission distance, active PMD compensation will have to be used.

PMD compensation is currently an active area of fiber-optic system research. In many adaptive PMD compensating systems [3], [4], as shown in Fig. 1(a), a polarization beam splitter (PBS) is used to separate the signals carried by the two PSPs. A polarization controller (PC) precedes the PBS to align the PSPs with the principal axes of the PBS. Following the PBS is a variable delay line to compensate for the link DGD. Finally, the two optical paths are recombined, and the effects of PMD can be entirely compensated in the optical domain. Continuous monitoring of the residual PMD can be derived from the signal to provide feedback signal parameters for controlling the $\mathrm{PC}$ and the variable delay line. A liquid-crystal-based PC is commercially available with small footprint (such as the E-TEK FPCR series), which provides endless polarization autotracking. However, the variable optical delay line in such a PMD compensator is often implemented using a mechanical system to provide the needed 

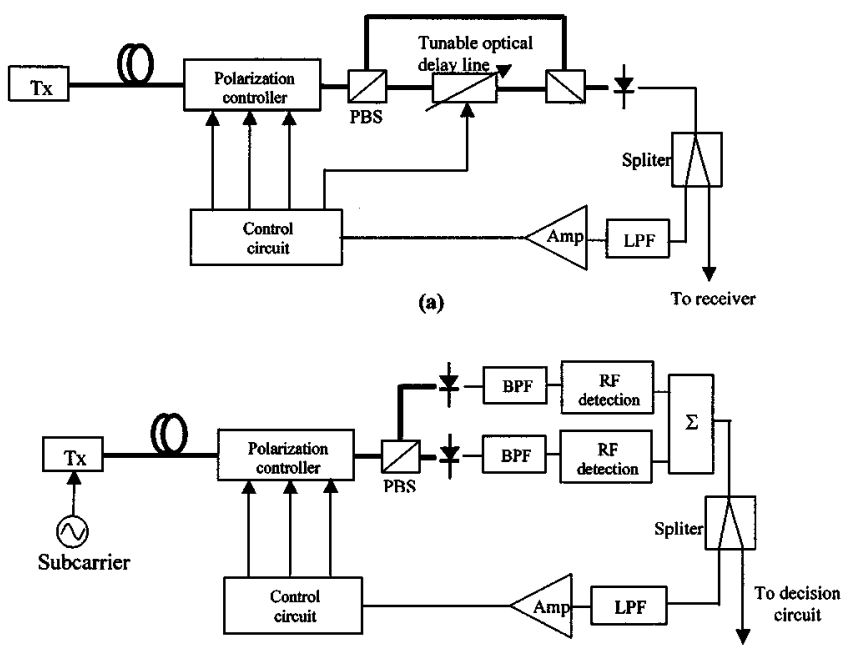

(b)

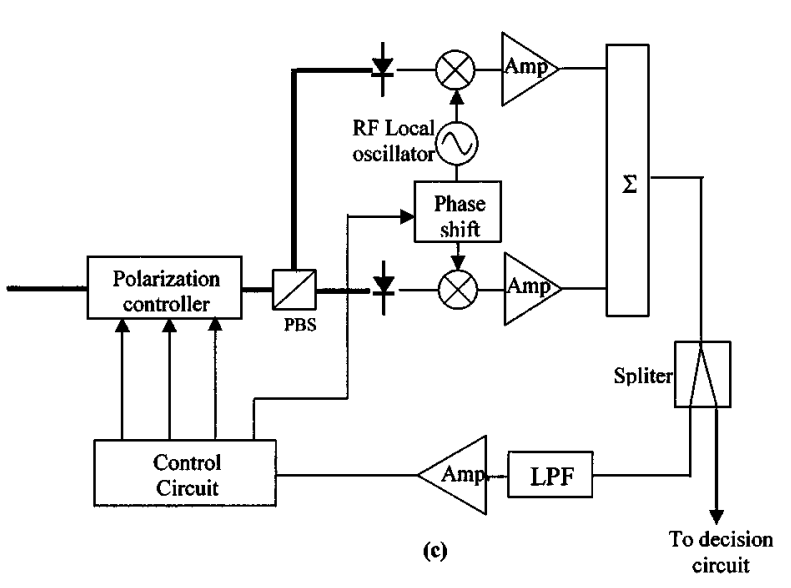

Fig. 1. Block diagrams. (a) Optical-domain PMD compensation using a tunable optical delay line. (b) PMD-insensitive SCM optical receiver using polarization diversity with RF envelope detection. (c) PMD-insensitive SCM optical receiver using polarization diversity with $\mathrm{RF}$-coherent detection.

DGD range. The speed, size, and reliability of this mechanism raise concerns.

Another PMD-compensating scheme in time-division-multiplexing (TDM) optical systems is to shift the tunable delay line to the electrical domain using a polarization diversity receiver [5]. In this method, a tunable RF delay line has to be used after one of the two photodiodes to correct the PMD-induced DGD. Compared with the optical compensation method shown in [3] and [4], the tunable RF delay line required in [5] is not necessarily easier to implement than is an optical delay line.

Since PMD-induced carrier fading is the major concern in SCM optical systems, we will show that polarization diversity optical receivers shown in Fig. 1(b) and (c) are effective in eliminating this carrier fading, making an RF delay line unnecessary. The setup shown in Fig. 1(b) works for amplitude shift keying (ASK) SCM modulation scheme. Two photodiodes are used to detect the two PSP components at the output of the system. In order to ensure the alignment between the principal axis of the PBS and the PSP of the fiber, a PC is used before the PBS. If the principal axis of the PBS is properly aligned with the PSPs of the optical fiber system at the carrier wavelength, the amplitude of signals detected by both photodiodes will not be affected by PMD-induced fading. The effect of PMD will be shown as

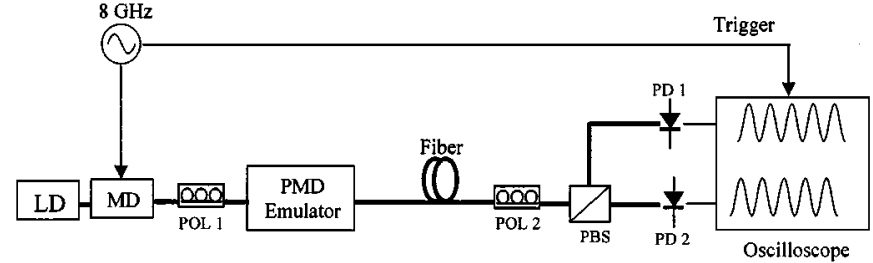

(a)

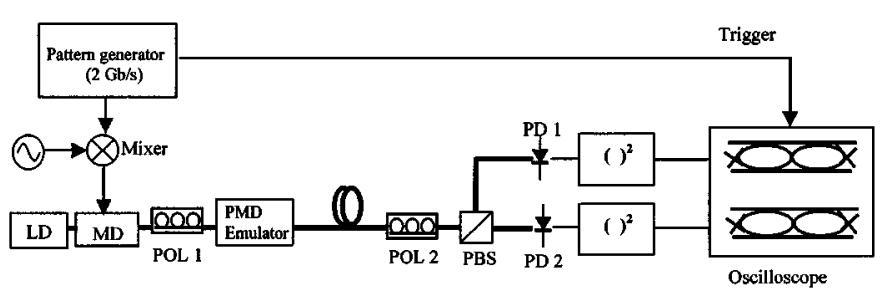

(b)

Fig. 2. Experimental setups. (a) An SCM system transmitting a nonmodulated 8-GHz subcarrier only. (b) An SCM system transmitting 2-Gb/s pseudorandom nonreturn to zero (NRZ) data carried on an 8-GHz subcarrier. LD: Laser diode. MD: Optical modulator. POL: PC. PBS: Polarization beam splitter. PD: Photodiode. ( $)^{2}$ : Square-law detector.

a relative time delay between the waveforms carried by the two PSP components. An RF bandpass filter is used after each photodiode to select the desired subcarrier channel, followed by an RF detector to detect the signal envelope and remove the high-frequency subcarrier. The signals carried by the two PSPs are recombined after the subcarrier is removed, and therefore, PMD-induced carrier fading is eliminated.

For phase-shift-keying-modulated SCM systems with coherent RF detection, an RF local oscillator is used to detect the baseband signal that is carried as phase information on each subcarrier component. To eliminate PMD-induced carrier fading in this type of system, a voltage-controlled phase shifter can be used, as shown in Fig. 1(c). Since PMD is a relatively slow process, the phase-tuning speed does not have to be very fast. This type of voltage controlled phase shifter is available commercially.

To verify this concept, we have first built a system transmitting only a subcarrier tone without any data on it. As shown in Fig. 2(a), in this experiment, an 8-GHz sinusoid was applied to an external optical modulator. A PMD emulator was used to create the desired amount of DGD. A PBS was used to separate the two orthogonal PSPs and a PC was used to align the principal axes of the PBS to the system PSPs. Two high-speed photodiodes were used to detect optical signals from both output arms of the PBS. A two-channel digital oscilloscope was used to display the detected signal waveforms.

With the DGD value of the PMD emulator set to zero, the two waveforms detected by both photodiodes are exactly in phase. Adjusting the angle of the PBS only resulted in amplitude redistribution between the two waveforms. By introducing a DGD using the PMD emulator, these two waveforms are no longer in phase, and the relative time delay between them is equal to the value of the DGD. Fig. 3 shows the measured waveforms when a fixed DGD is set at $62.5 \mathrm{ps}$, causing the two waveforms to be exactly out of phase. Fig. 3(a) was measured when the optical input is launched into the fiber with 50/50 splitting between the two PSPs and the principal axes of the PBS are aligned with the 

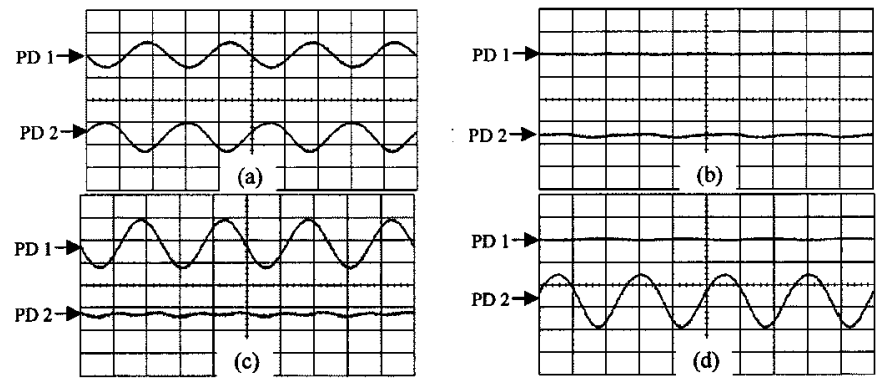

Fig. 3. Measured RF waveforms at the two photodiodes using the experimental setup shown in Fig. 3(a). Fiber system has 62.5-ps DGD. Horizontal scale: 50 ps/div. (a) PBS aligned with fiber PSPs; signal SOP is midway between the fast and the slow PSPs. (b) PBS is midway between the two fiber PSPs; the signal SOP is also midway between the two PSPs. (c) PBS is aligned with the fiber PSPs; the signal SOP is aligned with the fast PSP. (d) PBS is aligned with the fiber PSPs; the signal SOP is aligned with the slow PSP.

PSPs. In this case, the two waveforms have equal amplitude and opposite phase. Fig. 3(b) was obtained when the optical input was launched into the fiber with 50/50 splitting between the two PSPs, but the principal axes of the PBS is aligned halfway between the two PSPs. This is the worst case in terms of PMD effect, and complete carrier fading happens at both photodiodes.

Fig. 3(c) and (d) show the measured signal waveforms when the input optical signal is aligned with the fast and slow fiber PSPs, respectively. To avoid carrier fading at each diode, it is essential to align the principal axes of the PBS with the PSPs of the fiber system. In this case, since the optical-phase information is removed during photodetection, the sum of the RF signal power detected by the two photodiodes will be constant. The signal power partitioning in the two photodiodes will depend on the polarization alignment between laser source and the fiber PSPs.

In order to demonstrate the application of this concept in SCM digital systems, a digital transmission experiment was conducted using the setup shown in Fig. 2(b). In this experiment, a 2-Gb/s pseudorandom nonreturn-to-zero (NRZ) signal was carried by an 8-GHz RF subcarrier. Again, a 62.5-ps fixed DGD was artificially inserted by the PMD emulator. For simplicity, the fiber length between transmitter and receiver is short, and no chromatic dispersion is involved in the experiment. When the principal axes of the PBS are aligned with fiber system PSPs, no PMD distortion of signal waveforms results, but the relative amplitude of the waveforms detected by each photodiode depends on the signal state of polarization (SOP). Fig. 4(a) and (b) show the detected waveforms when the signal SOP is aligned with the fast and slow PSP, respectively, and Fig. 4(c) shows the waveforms when signal SOP is midway between the two PSPs. In this measurement, even though the amount of system DGD is $62.5 \mathrm{ps}$, which is equivalent to a $\pi$ phase shift of the RF carrier, the sum of the signal eye diagrams detected at the two receiver arms remains independent of the signal SOP. Because of the RF envelope detection after each photodiode, which eliminates the RF carrier, carrier fading is suppressed when combining the signal waveforms from the two branches.

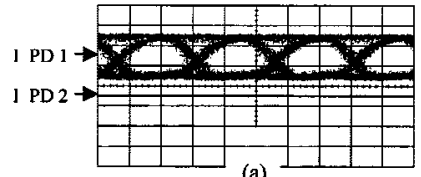

(a)

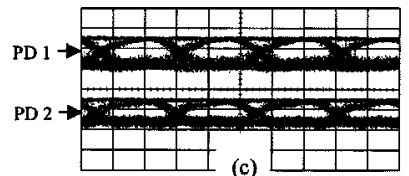

(c)

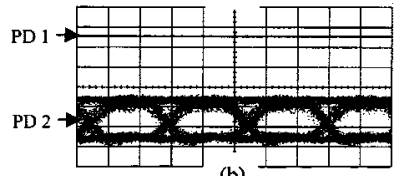

(b)

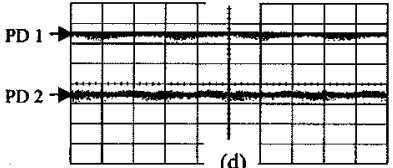

Fig. 4. Measured signal eye diagrams at the two photodiodes using the experimental setup shown in Fig. 3(b). Fiber system has 62.5-ps DGD. Horizontal scale: $200 \mathrm{ps} / \mathrm{div}$. (a) PBS aligned with the fiber PSPs; signal SOP aligned with the fast PSP. (b) PBS aligned with the fiber PSPs; signal SOP aligned with the slow PSP. (c) PBS aligned with the fiber PSPs; signal SOP is midway between the fast and the slow PSP. (d) PBS is midway between the two fiber PSPs; the signal SOP is also midway between the two PSPs.

On the other hand, if the principal axes of the PBS are not aligned with the fiber system PSPs, PMD-induced carrier fading would happen at both of the two detection arms. Fig. 4(d) shows the detected waveforms when the principal axis of the PBS is set midway between the two PSPs of the fiber. In this worst case, complete signal fading results.

It is important to note that there is no tunable delay line used in this receiver. Even though carrier fading can be avoided by removing RF phase information before adding signals from each photodiode, the relative delay between the two branches still exists, which is determined by the fiber system DGD. For typical SCM optical systems, where the data rate carried by each subcarrier is relatively low, a moderate amount of DGD will not significantly degrade system performance.

In conclusion, we have demonstrated a carrier-fading-free optical receiver for SCM optical systems using polarization diversity. Since a tunable optical delay line is not required in this setup, it may have advantages over optical-domain PMD compensation.

\section{ACKNOWLEDGMENT}

The authors would like to thank D. Richards for his useful comments.

\section{REFERENCES}

[1] R. Hui, B. Zhu, R. Huang, C. Allen, and K. Demarest, "High-speed optical transmission using subcarrier multiplexing," J. Lightwave Technol., vol. 20, pp. 417-427, Mar. 2002.

[2] O. H. Adamczyk, A. B. Sahin, Q. Yu, S. Lee, and A. E. Willner, "Statistics of PMD-induced power fading for double sideband and single sideband subcarrier-multiplexed signals," presented at the Tech. Dig. Opt. Fiber Commun. Conf., OFC'2001, Anaheim, CA, Mar. 19-22, Paper MO5.

[3] T. Takahashi, T. Imai, and M. Aiki, "Automatic compensation technique for timewise fluctuating polarization mode dispersion in in-line amplifier systems," Electron. Lett., vol. 30, pp. 348-349, 1994.

[4] H. Y. Pua, K. Peddanarappagari, B. Zhu, C. Allen, K. Demarest, and R. Hui, "An adaptive first-order polarization-mode dispersion compensation system aided by polarization scrambling: Theory and demonstration," J. Lightwave Technol., vol. 18, pp. 832-841, June 2000.

[5] B. W. Hakki, "Polarization mode dispersion compensation by phase diversity detection," Photon. Technol. Lett., vol. 9, pp. 121-123, Jan. 1997. 